\title{
Existence of Solutions for Unbounded Elliptic Equations with Critical Natural Growth
}

\author{
Aziz Bouhlal $\left(\mathbb{D},{ }^{1}\right.$ Abderrahmane El Hachimi $\left(\mathbb{D},{ }^{2}\right.$ Jaouad Igbida, ${ }^{3}$ \\ El Mostafa Sadek $\left(\mathbb{D},{ }^{4}\right.$ and Hamad Talibi Alaoui ${ }^{1}{ }^{1}$ \\ ${ }^{1}$ Labo Math Appli, Faculty of Sciences, B. P. 20, El Jadida, Morocco \\ ${ }^{2}$ Laboratory of Mathematical Analysis and Applications, Mohammed V University, Faculty of Sciences, Rabat, Morocco \\ ${ }^{3}$ Labo DGTIC, department of Mathematics, CRMEF El Jadida, Morocco \\ ${ }^{4}$ Laboratory LabSIPE, ENSA, d'EL Jadida, University Chouaib Doukkali, Morocco
}

Correspondence should be addressed to El Mostafa Sadek; sadek.maths@gmail.com

Received 29 June 2018; Accepted 26 October 2018; Published 26 November 2018

Academic Editor: Peiguang Wang

Copyright (C) 2018 Aziz Bouhlal et al. This is an open access article distributed under the Creative Commons Attribution License, which permits unrestricted use, distribution, and reproduction in any medium, provided the original work is properly cited.

We investigate existence and regularity of solutions to unbounded elliptic problem whose simplest model is $\left\{-\operatorname{div}\left[\left(1+|u|^{q}\right) \nabla u\right]+u=\right.$ $\gamma\left(|\nabla u|^{2} /(1+|u|)^{1-q}\right)+f$ in $\Omega, u=0$ on $\partial \Omega$, $\}$, where $0<q<1, \gamma>0$ and $f$ belongs to some appropriate Lebesgue space. We give assumptions on $f$ with respect to $q$ and $\gamma$ to show the existence and regularity results for the solutions of previous equation.

\section{Introduction}

In this paper, we consider the Dirichlet problem for some nonlinear elliptic problems such as

$$
\begin{aligned}
-\operatorname{div}\left(\left[a(x)+|u|^{q}\right] \nabla u\right)+u=H( & (x, u, \nabla u)+f, \\
& x \in \Omega, u \in H_{0}^{1}(\Omega),
\end{aligned}
$$

under the following assumptions: $\Omega$ is a bounded open subset of $\mathbb{R}^{N}$, where $N \geq 3,0<q<1$, and $f \in L^{m}$ with $m \geq 2$ and $a: \Omega \longrightarrow \mathbb{R}$ is a measurable function satisfying the following conditions:

$$
\alpha \leq a(x) \leq \beta,
$$

for almost every $x \in \Omega$, where $\alpha$ and $\beta$ are positive real constants. $H(x, s, \xi)$ is a Carathéodory-type function satisfying to:

$$
|H(x, s, \xi)| \leq \gamma \frac{|\xi|^{2}}{(1+|s|)^{1-q}}
$$

for some $\gamma>0$.
In [1], Arcoya, Boccardo, and Leonor obtained the existence and regularity results for the following elliptic problem with degenerate coercivity:

$$
-\operatorname{div}\left(\frac{\alpha \nabla u}{(1+|u|)^{2}}\right)+u=\gamma \frac{|\nabla u|^{2}}{(1+|u|)^{3}}+f
$$

$$
x \in \Omega, u \in H_{0}^{1}(\Omega),
$$

where $\alpha, \gamma>0, f \in L^{m}(\Omega)$ with $m \geq 2$, and $\Omega$ is a bounded subset of $\mathbb{R}^{N}, N \geq 3$.

The purpose of the present paper is to study the same kind of lower order terms as in problem (4) in the case of an elliptic operator with unbounded coefficients such as (1).

There are several papers concerned with existence and regularity of the solution for the following problem:

$$
\begin{aligned}
-\operatorname{div}(M(x, u) \nabla u)+g(x, u, \nabla u) & =f(x) \quad x \in \Omega, \\
u(x) & =0 \quad x \in \partial \Omega .
\end{aligned}
$$

We refer the intersting articles: Boccardo, Murat and Puel [2], Bensoussan, Boccardo and Murat [3], and Boccardo, Gallout [4]. In all these works $g$ is a nonlinear lower term having natural growth with respect to $\nabla u$, data $f$ in 
suitable Lebesgue spaces, and $M(x, u)$ is a Carathéodory-type bounded function subject to certain structural inequalities.

Another motivation for studying these problem arises from the calculus of variations in the case where $0 \leq f \in$ $L^{m}(\Omega)$ with $m \geq N / 2$ and

$$
g(x, u, \nabla u)=\frac{|\nabla u|^{2}}{u^{\theta}},
$$

where $\theta \in(0,1)$, which is considered by Puel in [5].

We point out that in [6] the authors considered $M(x, u)$ as a bounded function and

$$
g(x, u, \nabla u)=\frac{Q(x, u)|\nabla u|^{2}}{u^{\theta}},
$$

where $\theta \in(0,1]$. The function $Q(x, s): \Omega \times \mathbb{R} \longrightarrow \mathbb{R}^{N^{2}}$ is symmetric, measurable with respect to $x$ and continuous with respect to $s$ with the following uniform ellipticity condition: for $x \in \Omega$, and $s \in \mathbb{R}$,

$$
\mu|\xi|^{2} \leq \mathrm{Q}(x, s) \xi \xi \leq \nu|\xi|^{2}, \quad 0<\mu \leq \nu .
$$

We shall prove the following main results on existence and regularity of solutions for problem (1).

Theorem 1. Let $\widetilde{\alpha}=\min \{1, \alpha\}$. Assuming that the functions $a$ and $H$ satisfy (2) and (3) then, if $f$ belong to $L^{m}(\Omega)$, with

$$
m>2\left(\frac{\gamma}{\widetilde{\alpha}}+1\right)+q
$$

there exists a distributional solution $u \in W_{0}^{1,1}(\Omega)$ of problem (1) such that

$$
\begin{aligned}
& H(x, u, \nabla u) \in L^{1}(\Omega), \quad\left[a(x)+|u|^{q}\right]|\nabla u| \in L^{1}(\Omega), \\
& \int_{\Omega}\left[a(x)+|u|^{q}\right] \nabla u \nabla \psi+\int_{\Omega} u \psi \\
& =\int_{\Omega} H(x, u, \nabla u) \psi+\int_{\Omega} f \psi, \quad \forall \psi \in C_{0}^{\infty}(\Omega) .
\end{aligned}
$$

Furthermore, any solution of the problem (1) belongs to $H_{0}^{1}(\Omega)$.

In the next result, we consider the case where $f$ has a high summability.

Theorem 2. Let $\tilde{\alpha}=\min \{1, \alpha\}$, and assume that (2) and (3) hold true. If $u$ the solution given by Theorem 1 and $f$ belongs to $L^{m}(\Omega)$, with

$$
m>\max \left\{2\left(\frac{\gamma}{\tilde{\alpha}}+1\right)+q, \frac{N}{2}\left(\frac{\gamma}{\tilde{\alpha}}+1\right)\right\},
$$

then $u$ belongs to $H_{0}^{1}(\Omega) \cap L^{\infty}(\Omega)$.

The rest of the paper is organized as follows: Section 2 is devoted to give some a priori estimates for the approximated problem associated with problem (1); while in Section 3, we give the detailed proofs of Theorems 1 and 2 .

\section{The Approximated Problem}

In this section, we use the hypotheses (2) and (3) and we suppose that

$$
\widetilde{\alpha}(m-1)-\gamma>0,
$$

where $\tilde{\alpha}=\min \{1, \alpha\}$ holds true. To prove Theorem 1 and Theorem 2, we will use the following approximating problems associated with problem (1):

$$
\begin{aligned}
& -\operatorname{div}\left(\left[a(x)+\left|u_{n}\right|^{q}\right] \nabla u_{n}\right)+u_{n} \\
& =H_{n}\left(x, u_{n}, \nabla u_{n}\right)+f_{n}, \quad x \in \Omega,
\end{aligned}
$$

where

$$
f_{n}(x)=\frac{f(x)}{1+(1 / n)|f(x)|}
$$

and

$$
H_{n}(x, s, \xi)=\frac{H(x, s, \xi)}{1+(1 / n)|\xi|^{2}} .
$$

By the results of $[2,4]$ there exists a weak solution $u_{n}$ in $H_{0}^{1}(\Omega) \cap L^{\infty}(\Omega)$ of problem (13) in the sense that

$$
\begin{array}{r}
\int_{\Omega}\left[a(x)+\left|u_{n}\right|^{q}\right] \nabla u_{n} \nabla \varphi+\int_{\Omega} u_{n} \varphi \\
=\int_{\Omega} H_{n}\left(x, u_{n}, \nabla u_{n}\right) \varphi+\int_{\Omega} f_{n} \varphi
\end{array}
$$

for every $\varphi \in H_{0}^{1}(\Omega) \cap L^{\infty}(\Omega)$.

The following lemma will be very useful, as it gives us an a priori estimate on the summability of the solutions to problems (13).

Lemma 3. If $u_{n}$ is a solution to problem (13), then for every $k \geq 0$,

$$
\int_{\Omega}\left|G_{k}\left(u_{n}\right)\right|^{m} \leq \int_{\left\{\left|u_{n}\right| \geq k\right\}}|f|^{m}
$$

Moreover, there exist $R>0$ depending on $\|f\|_{L^{m}(\Omega)}, \alpha, q$, and $\gamma$, such that

$$
\left\|u_{n}\right\|_{H_{0}^{1}(\Omega)} \leq R .
$$

Remark 4. (i) Let $\left\{u_{n}\right\}$ be a sequence of solutions $u_{n}$ of (13). As a consequence of Lemma 3 , there exists $u \in H_{0}^{1}(\Omega)$ such that, up to a subsequence, $u_{n}$ converges weakly to $u$ in $H_{0}^{1}(\Omega)$ and a.e. in $\Omega$.

(ii) By the previous lemma we deduce from (3) that

$$
\left\|H_{n}\left(x, u_{n}, \nabla u_{n}\right)\right\|_{L^{1}(\Omega)} \leq \gamma \int_{\Omega} \frac{\left|\nabla u_{n}\right|^{2}}{\left(1+\left|u_{n}\right|\right)^{1-q}} \leq \gamma R^{2} .
$$

Proof of Lemma 3. In order to prove (17), we claim that by assumption (2) and $q<1$, there exist positive constant $c_{0}$ such that

$$
\widetilde{\alpha}(1+|t|)^{q} \leq a(x)+|t|^{q} \leq c_{0}(1+|t|)^{q}, \quad \forall t \in \mathbb{R} .
$$


Choosing $\varphi=\left|G_{k}\left(u_{n}\right)\right|^{m-1} \operatorname{sgn}\left(u_{n}\right)$ in (16) and using (20), we obtain

$$
\begin{aligned}
\tilde{\alpha}(m-1) & \int_{\Omega}\left(1+\left|u_{n}\right|\right)^{q}\left|\nabla u_{n}\right|^{2}\left|G_{k}\left(u_{n}\right)\right|^{m-2} \\
& +\int_{\Omega}\left|u_{n}\right|\left|G_{k}\left(u_{n}\right)\right|^{m-1} \\
\leq & \gamma \int_{\Omega} \frac{\left|\nabla u_{n}\right|^{2}}{\left(1+\left|u_{n}\right|\right)^{1-q}}\left|G_{k}\left(u_{n}\right)\right|^{m-1} \\
& +\int_{\Omega}\left|f_{n}\right|\left|G_{k}\left(u_{n}\right)\right|^{m-1} .
\end{aligned}
$$

Thus, joining the terms involving the gradient, we get

$$
\begin{gathered}
\int_{\Omega}\left[\tilde{\alpha}(m-1)-\gamma \frac{\left|G_{k}\left(u_{n}\right)\right|}{1+\left|u_{n}\right|}\right]\left(1+\left|u_{n}\right|\right)^{q}\left|\nabla u_{n}\right|^{2} \\
\cdot\left|G_{k}\left(u_{n}\right)\right|^{m-2}+\int_{\Omega}\left|G_{k}\left(u_{n}\right)\right|^{m} \leq \int_{\Omega}\left|f_{n}\right| \\
\cdot\left|G_{k}\left(u_{n}\right)\right|^{m-1} .
\end{gathered}
$$

Using (12) we deduce that

$$
\int_{\Omega}\left|G_{k}\left(u_{n}\right)\right|^{m} \leq \int_{\Omega}|f|\left|G_{k}\left(u_{n}\right)\right|^{m-1},
$$

and the Hölder inequality on the right hand side yields

$$
\begin{aligned}
& \int_{\Omega}\left|G_{k}\left(u_{n}\right)\right|^{m} \\
& \quad \leq\left(\int_{\left\{\left|u_{n}\right| \geq k\right\}}|f|^{m}\right)^{1 / m}\left(\int_{\Omega}\left|G_{k}\left(u_{n}\right)\right|^{m}\right)^{1-1 / m},
\end{aligned}
$$

which implies (17).

Let us choose now $\varphi=\left[\left(1+\left|u_{n}\right|\right)^{m-1}-1\right] \operatorname{sgn}\left(u_{n}\right)$ as a test function in (16), and we obtain

$$
\begin{gathered}
(\widetilde{\alpha}(m-1)-\gamma) \int_{\Omega}\left(1+\left|u_{n}\right|\right)^{m-2+q}\left|\nabla u_{n}\right|^{2} \\
\leq\|f\|_{L^{m}(\Omega)}\left(\int_{\Omega}\left(1+\left|u_{n}\right|\right)^{m}\right)^{1-1 / m} .
\end{gathered}
$$

Since $m \geq 2$, the previous calculations imply

$$
\int_{\Omega}\left|\nabla u_{n}\right|^{2} \leq c\left(\int_{\Omega}\left(1+\left|u_{n}\right|\right)^{m}\right)^{1-1 / m} .
$$

Using (17) with $k=0$, (18) follows.

Lemma 5. Let $u_{n}$ be the sequence of solutions to problems (13) and let the function $u$ given by Remark 4 . Then $u_{n}$ strongly converges to $u$ in $L^{m}(\Omega)$. Moreover $\nabla u_{n}$ strongly converges to $\nabla u$ in $L^{1}(\Omega)^{N}$.

Remark 6. Note that (25) implies that there exists $\delta>0$ independent of $n$ such that

$$
\int_{\Omega}\left(1+\left|u_{n}\right|\right)^{m-2+q}\left|\nabla u_{n}\right|^{2} \leq \delta .
$$

By using the previous lemma, we deduce that

$$
\begin{array}{r}
\left(1+\left|u_{n}\right|\right)^{(m-2+q) / 2}\left|\nabla u_{n}\right| \longrightarrow(1+|u|)^{(m-2+q) / 2}|\nabla u| \\
\text { weakly in } L^{2}(\Omega)^{N} .
\end{array}
$$

Proof of Lemma 5. We use (17) written for $k=0$ :

$$
\int_{\Omega}\left|u_{n}\right|^{m} \leq \int_{\Omega}|f|^{m} \leq c .
$$

Since $u_{n}$ almost everywhere converges to $u$, we have from Fatou's lemma that

$$
\int_{\Omega}|u|^{m} \leq c
$$

Hence $u$ belongs to $L^{m}(\Omega)$. Using assumption (17), for any $k>0$ we have

$$
\begin{aligned}
\int_{E}\left|u_{n}\right|^{m} & \leq \int_{E \cap\left\{\left|u_{n}\right| \leq k\right\}}\left|u_{n}\right|^{m}+\int_{E \cap\left\{\left|u_{n}\right| \geq k\right\}}\left|u_{n}\right|^{m} \\
& \leq k^{m} \operatorname{meas}(E)+\int_{\left\{\left|u_{n}\right| \geq k\right\}}|f|^{m} .
\end{aligned}
$$

As before, we first choose $k$ such that the second integral is small, uniformly with respect to $n$, and then the measure of $E$ small enough such that the first term is small. The almost everywhere convergence of $u_{n}$ to $u$ and Vitali's theorem imply that $u_{n}$ strongly converges to $u$ in $L^{m}(\Omega)$.

For the second convergence, we will follow the same technique as in [1] (see also [7]). Let $h, k>0$. In the sequel $C$ will denote a constant independent of $n, h, k$. Let us consider $T_{h}\left[u_{n}-T_{k}(u)\right]$ as a test function in problems (16). Then,

$$
\begin{aligned}
& \int_{\Omega}\left[a(x)+\left|u_{n}\right|^{2}\right] \nabla u_{n} \nabla T_{h}\left[u_{n}-T_{k}(u)\right] \\
& \quad+\int_{\Omega} u_{n} T_{h}\left[u_{n}-T_{k}(u)\right] \\
& \leq\left(\|f\|_{L^{1}(\Omega)}+\left\|H_{n}\left(x, u_{n}, \nabla u_{n}\right)\right\|_{L^{1}(\Omega)}\right) h .
\end{aligned}
$$

Moreover, thanks to the $L^{m}(\Omega)$ convergence of $u_{n}$, the second integral in (32) converges (as n diverges) to a positive number. Thus, it yields to

$$
\begin{aligned}
& \alpha \int_{\Omega}\left|\nabla T_{h}\left[u_{n}-T_{k}(u)\right]\right|^{2} \\
& \leq\left(\|f\|_{L^{1}(\Omega)}+\gamma R^{2}\right) h \\
& \quad-\int_{\Omega}\left[a(x)+\left|u_{n}\right|^{q}\right] \nabla T_{k}(u) \nabla T_{h}\left[u_{n}-T_{k}(u)\right] .
\end{aligned}
$$

Let $\mathscr{K}=h+k$, observing that $\nabla T_{h}\left[u_{n}-T_{k}(u)\right]=0$ if $\left|u_{n}\right|>\mathscr{K}$, then

$$
\begin{aligned}
& \int_{\Omega}\left[a(x)+\left|u_{n}\right|^{q}\right] \nabla T_{k}(u) \nabla T_{h}\left[u_{n}-T_{k}(u)\right] \\
& =\int_{\Omega}\left[a(x)+\left|T_{\mathscr{K}}\left(u_{n}\right)\right|^{q}\right] \nabla T_{k}(u) \nabla T_{h}\left[u_{n}-T_{k}(u)\right] .
\end{aligned}
$$


Since $T_{h}\left[u_{n}-T_{k}(u)\right]$ converges to $T_{h}\left[u-T_{k}(u)\right]$ weakly in $\left(L^{2}(\Omega)\right)^{N}$ and $\left[a(x)+\left|T_{\mathscr{K}}\left(u_{n}\right)\right|^{q}\right] \nabla T_{k}(u)$ strongly converges to $\left[a(x)+\left|T_{\mathscr{K}}(u)\right|^{q}\right] \nabla T_{k}(u)$ in $\left(L^{2}(\Omega)\right)^{N}$, we have

$$
\begin{aligned}
& \lim _{n \longrightarrow+\infty} \int_{\Omega}\left[a(x)+\left|u_{n}\right|^{q}\right] \nabla T_{k}(u) \nabla T_{h}\left[u_{n}-T_{k}(u)\right] \\
& \quad=0
\end{aligned}
$$

thus, yielding

$$
\int_{\Omega}\left|\nabla T_{h}\left[u_{n}-T_{k}(u)\right]\right|^{2} \leq C h+\varepsilon(n),
$$

where $\varepsilon(n)$ denote any quantity that vanishes as $n$ diverges. Hence, by Hölder's inequality, we deduce that

$$
\begin{aligned}
& \int_{\left\{\left|u_{n}-u\right| \leq h,|u| \leq k\right\}}\left|\nabla\left(u_{n}-u\right)\right|=\int_{\Omega}\left|\nabla T_{h}\left[u_{n}-T_{k}(u)\right]\right| \\
& \quad \leq|\Omega|^{1 / 2} \sqrt{C h+\varepsilon(n)} .
\end{aligned}
$$

Fix, now, $\epsilon>0$ there exist $h_{0}$ such that, for $h<h_{0}$, we have

$$
|\Omega|^{1 / 2} \sqrt{C h}<\epsilon
$$

Thanks to the weak convergence of $u_{n}$ in $H_{0}^{1}(\Omega)$ and the absolute continuity of the integral, there exists $k_{0}$ independent from $n$ such that, for $k>k_{0}$, we have

$$
\int_{\{|u|>k\}}\left|\nabla u_{n}\right|+\int_{\{|u|>k\}}|\nabla u| \leq \epsilon .
$$

In addition, by Dunford Pettis Theorem, we deduce that there exists $n(h, \epsilon)$ such that, for $n>n(h, \epsilon)$, we have

$$
\int_{\left\{\left|u_{n}-u\right|>h\right\}}\left|\nabla\left(u_{n}-u\right)\right| \leq \epsilon .
$$

We can write

$$
\begin{aligned}
\int_{\Omega}\left|\nabla\left(u_{n}-u\right)\right|= & \int_{\left\{\left|u_{n}-u\right| \leq h,|u| \leq k\right\}}\left|\nabla\left(u_{n}-u\right)\right| \\
& +\int_{\left\{\left|u_{n}-u\right| \leq h,|u|>k\right\}}\left|\nabla\left(u_{n}-u\right)\right| \\
& +\int_{\left\{\left|u_{n}-u\right|>h\right\}}\left|\nabla\left(u_{n}-u\right)\right| .
\end{aligned}
$$

Using (37), (39), and (40), for $h<h_{0}$ and $n>n(h, \epsilon)$, we have

$$
\int_{\Omega}\left|\nabla\left(u_{n}-u\right)\right| \leq 3 \epsilon+\varepsilon(n) .
$$

This proves the strong convergence of $\nabla u_{n}$ to $\nabla u$ in $L^{1}(\Omega)^{N}$. $\left\{u_{n}\right\}$.

The following lemma yields some a priori estimate on

Lemma 7. Let $u$ be the function given by Remark 4 . Then $|u|^{q}|\nabla u|$ belongs to $L^{r}(\Omega)$, for every $r<N /(N-1)$.
Proof. For every $\lambda>1$, we take $\left[1-1 /\left(1+\left|u_{n}\right|\right)^{\lambda-1}\right] \operatorname{sign}\left(u_{n}\right)$ as a test function in (16). Droping positive terms yields

$$
\begin{aligned}
& \tilde{\alpha}(\lambda-1) \int_{\Omega} \frac{\left(1+\left|u_{n}\right|^{q}\right)\left|\nabla u_{n}\right|^{2}}{\left(1+\left|u_{n}\right|\right)^{\lambda}} \\
& \quad \leq\|f\|_{L^{1}(\Omega)}+\left\|H_{n}\left(x, u_{n}, \nabla u_{n}\right)\right\|_{L^{1}(\Omega)} .
\end{aligned}
$$

Hence, using $q<1$, it follows that

$$
\int_{\Omega} \frac{\left|\nabla u_{n}\right|^{2}}{\left(1+\left|u_{n}\right|\right)^{\lambda-q}} \leq \frac{\|f\|_{L^{1}(\Omega)}+\gamma R^{2}}{\widetilde{\alpha}(\lambda-1)} .
$$

On the other hand, for every $\lambda>1$; we have

$$
\begin{aligned}
& \int_{\Omega}\left|u_{n}\right|^{q r}\left|\nabla u_{n}\right|^{r} \\
& \leq \int_{\Omega} \frac{\left|\nabla u_{n}\right|^{r}}{\left(1+\left|u_{n}\right|\right)^{r(\lambda-q) / 2}}\left(1+\left|u_{n}\right|\right)^{r(\lambda+q) / 2}, \\
& \leq\left(\frac{\|f\|_{L^{1}(\Omega)}+\gamma R^{2}}{\widetilde{\alpha}(\lambda-1)}\right)^{r / 2} \\
& \cdot\left(\int_{\Omega}\left(1+\left|u_{n}\right|\right)^{r(\lambda+q) /(2-r)}\right)^{(2-r) / 2} .
\end{aligned}
$$

Then, we obtain

$$
\left(\int_{\Omega}\left|u_{n}\right|^{(q+1) r^{*}}\right)^{r / r^{*}} \leq c\left(\int_{\Omega}\left|u_{n}\right|^{r(\lambda+q) /(2-r)}\right)^{(2-r) / 2} .
$$

Let us choose $r$ such that $(q+1) r^{*}=r(\lambda+q) /(2-r)$, that is

$$
r=\frac{N(2+q-\lambda)}{N(q+1)-(\lambda+q)}
$$

Since $\lambda>1$, we then have an estimate on $\left|u_{n}\right|^{q}\left|\nabla u_{n}\right|$ in $L^{r}(\Omega)$, for every $r<N /(N-1)$.

The next result will be used in the proof of Theorem 2 .

Lemma 8. Suppose that (2), (3), and (11) hold true. Let $f \in$ $L^{m}(\Omega)$ and $\left\{u_{n}\right\}$ be a solution of (13) with $f_{n}=f$ for every $n \epsilon$ $\mathbb{N}$. Then the norms of $\left\{u_{n}\right\}$ in $L^{\infty}(\Omega)$ and in $H_{0}^{1}(\Omega)$ are bounded by a constant which depends on $q, m, N, \alpha, \gamma$, meas $(\Omega)$ and on the norm of $f$ in $L^{m}(\Omega)$.

Proof. Since $m>(N / 2)(\gamma / \widetilde{\alpha}+1)$, we have $(1 / 2)(\gamma / \widetilde{\alpha}+1)<$ $m / N$. Let us choose $\sigma>0$ such that

$$
\frac{1}{2}\left(\frac{\gamma}{\widetilde{\alpha}}+q+1\right)<\sigma<\frac{m}{N}+\frac{q}{2} .
$$

The use of

$$
\left[\left(1+\left|u_{n}\right|\right)^{2 \sigma-q-1}-(1+k)^{2 \sigma-q-1}\right]^{+} \operatorname{sign}\left(u_{n}\right),
$$


as test function in (16), (3), and (20), implies that

$$
\begin{aligned}
& (2 \sigma-q-1) \tilde{\alpha} \int_{A_{k}}\left|\nabla u_{n}\right|^{2}\left(1+\left|u_{n}\right|\right)^{2 \sigma-2} \\
& \quad+\int_{A_{k}}\left|u_{n}\right|\left[\left(1+\left|u_{n}\right|\right)^{2 \sigma-q-1}-(1+k)^{2 \sigma-q-1}\right] \\
& \leq \gamma \int_{A_{k}} \frac{\left|\nabla u_{n}\right|^{2}}{\left(1+\left|u_{n}\right|\right)^{1-q}}\left(1+\left|u_{n}\right|\right)^{2 \sigma-q-1} \\
& \quad+\int_{A_{k}}\left|f_{n}\right|\left(1+\left|u_{n}\right|\right)^{2 \sigma-q-1},
\end{aligned}
$$

where

$$
A_{k}=\left\{x \in \Omega:\left|u_{n}\right|>k\right\} .
$$

By Young and Hölder's inequalities, we find

$$
\begin{aligned}
& {[(2 \sigma-q-1) \tilde{\alpha}-\gamma] \int_{A_{k}}\left|\nabla u_{n}\right|^{2}\left(1+\left|u_{n}\right|\right)^{2 \sigma-2}} \\
& \quad \leq C_{1} \int_{A_{k}}\left(1+\left|u_{n}\right|\right)^{2 \sigma-q}+C_{2} \int_{A_{k}}|f|^{2 \sigma-q} \\
& \quad \leq C_{m}\left(\operatorname{meas} A_{k}\right)^{1-(2 \sigma-q) / m} .
\end{aligned}
$$

Then, using Sobolev's inequality gives

$$
\begin{aligned}
& {[(2 \sigma-q-1) \tilde{\alpha}-\gamma]} \\
& \quad \cdot \frac{\mathcal{S}^{2}}{\sigma^{2}}\left(\int_{A_{k}}\left[\left(1+\left|u_{n}\right|\right)^{\sigma}-(1+k)^{\sigma}\right]^{2^{*}}\right)^{2 / 2^{*}} \\
& \quad \leq C_{m}\left(\operatorname{meas}_{k}\right)^{1-(2 \sigma-q) / m},
\end{aligned}
$$

where $\mathcal{S}$ denotes the best constant in Sobolev inequality. Now, we set

$$
\left(1+\left|u_{n}\right|\right)^{\sigma}=v_{n}
$$

and

$$
(1+k)^{\sigma}=h
$$

and the fact that $A_{k}=\left\{x \in \Omega: v_{n}>h\right\}$., the last inequality gives

$$
\begin{aligned}
& {[(2 \sigma-q-1) \tilde{\alpha}-\gamma] \frac{\mathcal{S}^{2}}{\sigma^{2}}\left(\int_{A_{k}}\left(v_{n}-h\right)^{2^{*}}\right)^{2 / 2^{*}}} \\
& \quad \leq C_{m}\left({\text { meas } \left.A_{k}\right)^{1-(2 \sigma-q) / m} .} .\right.
\end{aligned}
$$

Note that $\sigma<m / N+q / 2$ implies that $[1-(2 \sigma-$ $q) / m]\left(2^{*} / 2\right)>1$. Then Stampacchia's technique implies the following relation for some positive constant $C_{3}$,

$$
\left\|v_{n}\right\|_{L^{\infty}(\Omega)}=\left\|\left(1+\left|u_{n}\right|\right)^{\sigma}\right\|_{L^{\infty}(\Omega)} \leq C_{3},
$$

that is, $\left\|u_{n}\right\|_{L^{\infty}(\Omega)}$ is bounded.

\section{Proof of the Main Results}

We are now ready to prove the main result of this paper. We first observe that condition (9) implies (12). Hence the results of the previous section hold true. In order to prove the result, we have to pass to the limit in (16). To this aim, let $g$ be a function in $C^{1}(\mathbb{R})$ such that

$$
g(s)= \begin{cases}\frac{1+s}{\widetilde{\alpha} \rho-\gamma} & \text { if } s \geq 0 \\ \frac{1}{(1-s)(\widetilde{\alpha} \rho-\gamma)} & \text { if } s<0,\end{cases}
$$

where

$$
\rho=\frac{m-q-2}{2}
$$

Observe that, by (9), $g$ is positive, increasing, and it verifies

$$
\tilde{\alpha} \rho g^{\prime}(s)-\gamma \frac{g(s)}{1+|s|}>0, \quad \forall s \in \mathbb{R} \text {. }
$$

We will use, for $k>0$ and $s \in \mathbb{R}$,

$$
R_{k}(s)=1-T_{1}\left(G_{k}(s)\right),
$$

to define a test function. Remark that $R_{k} \geq 0,-k-1 \leq R_{k}(s) \leq$ $k+1$ and

$$
R_{k}^{\prime}(s)= \begin{cases}1 & \text { if }-k-1 \leq s \leq-k \\ -1 & \text { if } k \leq s \leq k+1 \\ 0 & \text { otherwise. }\end{cases}
$$

First of all, note that the a.e. convergence of $\nabla u_{n}$ (see Lemma 5), Remark 6, and (20) imply both that

$$
\left[a(x)+\left|u_{n}\right|^{q}\right] g^{\rho}\left(u_{n}\right) \nabla u_{n} \longrightarrow
$$

$$
\left[a(x)+|u|^{q}\right] g^{\rho}(u) \nabla u \quad \text { weakly in } L^{2}(\Omega)^{N}
$$

and

$$
\begin{gathered}
{\left[a(x)+\left|u_{n}\right|^{q}\right] \frac{1}{g^{\rho}\left(u_{n}\right)} \nabla u_{n} \longrightarrow} \\
{\left[a(x)+|u|^{q}\right] \frac{1}{g^{\rho}(u)} \nabla u \quad \text { weakly in } L^{2}(\Omega)^{N},}
\end{gathered}
$$

where $\rho$ is defined in (59).

The proof of the result will be achieved in two steps.

Step 1 (The first inequality). We fix $\psi \in H_{0}^{1}(\Omega) \cap L^{\infty}(\Omega)$, with $\psi \geq 0$, and take

$$
\phi=\frac{g^{\rho}\left(u_{n}\right)}{g^{\rho}(u)} R_{k}(u) \psi
$$


As test function in (16), we have that

$$
\begin{aligned}
& \int_{\Omega}\left[a(x)+\left|u_{n}\right|^{q}\right] \nabla u_{n} \nabla \psi \frac{g^{\rho}\left(u_{n}\right)}{g^{\rho}(u)} R_{k}(u) \\
& -\rho \int_{\Omega}\left[a(x)+\left|u_{n}\right|^{q}\right] \nabla u_{n} \nabla u \frac{g^{\rho}\left(u_{n}\right)}{g^{\rho+1}(u)} g^{\prime}(u) R_{k}(u) \\
& \cdot \psi+\int_{\Omega}\left[a(x)+\left|u_{n}\right|^{q}\right] \nabla u_{n} \nabla u \frac{g^{\rho}\left(u_{n}\right)}{g^{\rho}(u)} R_{k}^{\prime}(u) \psi \\
& +\rho \int_{\Omega}\left[a(x)+\left|u_{n}\right|^{q}\right] \nabla u_{n} \nabla u_{n} \frac{g^{\rho-1}\left(u_{n}\right)}{g^{\rho}(u)} g^{\prime}\left(u_{n}\right) \\
& \cdot R_{k}(u) \psi-\int_{\Omega} H_{n}\left(x, u_{n}, \nabla u_{n}\right) \frac{g^{\rho}\left(u_{n}\right)}{g^{\rho}(u)} R_{k}(u) \psi \\
& +\int_{\Omega} u_{n} \frac{g^{\rho}\left(u_{n}\right)}{g^{\rho}(u)} R_{k}(u) \psi=\int_{\Omega} f_{n} \frac{g^{\rho}\left(u_{n}\right)}{g^{\rho}(u)} R_{k}(u) \psi
\end{aligned}
$$

Remark now that, by the assumptions on $a, H$, relation (60) and the fact that $\psi \geq 0$, then we have

$$
\begin{gathered}
\rho\left[a(x)+\left|u_{n}\right|^{q}\right] \nabla u_{n} \nabla u_{n} \frac{g^{\rho-1}\left(u_{n}\right)}{g^{\rho}(u)} g^{\prime}\left(u_{n}\right) R_{k}(u) \psi \\
-H_{n}\left(x, u_{n}, \nabla u_{n}\right) \frac{g^{\rho}\left(u_{n}\right)}{g^{\rho}(u)} R_{k}(u) \psi \geq\left(1+\left|u_{n}\right|\right)^{q} \\
\cdot\left|\nabla u_{n}\right|^{2} \frac{g^{\rho-1}\left(u_{n}\right)}{g^{\rho}(u)} R_{k}(u) \\
\cdot \psi\left[\tilde{\alpha} \rho g^{\prime}\left(u_{n}\right)-\gamma \frac{g\left(u_{n}\right)}{1+\left|u_{n}\right|}\right] \geq 0 .
\end{gathered}
$$

Therefore, using the almost everywhere convergence of both $\nabla u_{n}$ and $u_{n}$, and applying Fatou's lemma, we get

$$
\begin{aligned}
& \liminf _{n \rightarrow \infty} \rho \int_{\Omega}\left[a(x)+\left|u_{n}\right|^{q}\right] \nabla u_{n} \nabla u_{n} \frac{g^{\rho-1}\left(u_{n}\right)}{g^{\rho}(u)} g^{\prime}\left(u_{n}\right) \\
& \cdot R_{k}(u) \psi-\int_{\Omega} H_{n}\left(x, u_{n}, \nabla u_{n}\right) \frac{g^{\rho}\left(u_{n}\right)}{g^{\rho}(u)} R_{k}(u) \psi \\
& \quad \geq \rho \int_{\Omega}\left[a(x)+|u|^{q}\right] \nabla u \nabla u \frac{g^{\prime}(u)}{g(u)} R_{k}(u) \psi \\
& -\int_{\Omega} H(x, u, \nabla u) R_{k}(u) \psi
\end{aligned}
$$

Furthermore, by using Lebesgue's theorem and (63), we obtain

$$
\begin{gathered}
\lim _{n \rightarrow \infty} \int_{\Omega}\left[a(x)+\left|u_{n}\right|^{q}\right] \nabla u_{n} \nabla \psi \frac{g^{\rho}\left(u_{n}\right)}{g^{\rho}(u)} R_{k}(u) \\
\quad=\int_{\Omega}\left[a(x)+|u|^{q}\right] \nabla u \nabla \psi R_{k}(u),
\end{gathered}
$$

and

$$
\begin{gathered}
\lim _{n \longrightarrow \infty} \rho \int_{\Omega}\left[a(x)+\left|u_{n}\right|^{q}\right] \nabla u_{n} \nabla u \frac{g^{\rho}\left(u_{n}\right)}{g^{\rho+1}(u)} g^{\prime}(u) R_{k}(u) \\
\cdot \psi=\rho \int_{\Omega}\left[a(x)+|u|^{q}\right] \nabla u \nabla u \frac{g^{\prime}(u)}{g(u)} R_{k}(u) \psi .
\end{gathered}
$$

Similarly, using the convergence $\left(u_{n}-f_{n}\right) \longrightarrow(u-f)$ in $L^{m}(\Omega)$, we have

$$
\begin{gathered}
\lim _{n \rightarrow \infty} \int_{\Omega}\left(u_{n}-f_{n}\right) \frac{g^{\rho}\left(u_{n}\right)}{g^{\rho}(u)} R_{k}(u) \psi \\
\quad=\int_{\Omega}(u-f) R_{k}(u) \psi
\end{gathered}
$$

Now, from (62), we get

$$
\begin{aligned}
& \lim _{n \rightarrow \infty} \int_{\Omega}\left[a(x)+\left|u_{n}\right|^{q}\right] \nabla u_{n} \nabla u \frac{g^{\rho}\left(u_{n}\right)}{g^{\rho}(u)} R_{k}^{\prime}(u) \psi \\
& \quad=\int_{\Omega}\left[a(x)+|u|^{q}\right] \nabla u \nabla u R_{k}^{\prime}(u) \\
& \quad=\int_{\{k \leq|u| \leq k+1\}}\left[a(x)+|u|^{q}\right] \nabla u \nabla u .
\end{aligned}
$$

Passing to the limit in (66) when $n$ tends to infinity and gathering together (68)-(72), weobtain

$$
\begin{aligned}
\int_{\Omega}\left[a(x)+|u|^{q}\right] \nabla u \nabla \psi R_{k}(u)+\int_{\Omega} u R_{k}(u) \psi \\
\quad+\int_{\{k \leq|u| \leq k+1\}}\left[a(x)+|u|^{q}\right] \nabla u \nabla u \\
\quad-\int_{\Omega} H(x, u, \nabla u) R_{k}(u) \psi \leq \int_{\Omega} f R_{k}(u) \psi .
\end{aligned}
$$

Choosing $T_{1}\left(G_{k}\left(u_{n}\right)\right)$ in (16), we get

$$
\begin{aligned}
& \int_{\left\{k \leq\left|u_{n}\right| \leq k+1\right\}}\left[a(x)+\left|u_{n}\right|^{q}\right] \nabla u_{n} \nabla u_{n} \\
& \quad \leq \int_{\left\{k \leq\left|u_{n}\right|\right\}}|f|+\int_{\left\{k \leq\left|u_{n}\right|\right\}}\left|H\left(x, u_{n}, \nabla u_{n}\right)\right| \\
& \quad \leq \int_{\left\{k \leq\left|u_{n}\right|\right\}}|f|+\frac{\gamma R^{2}}{(1+k)^{1-q}} .
\end{aligned}
$$

By Fatou's lemma, we have

$$
\lim _{k \rightarrow \infty} \int_{\{k \leq|u| \leq k+1\}}\left[a(x)+|u|^{q}\right] \nabla u \nabla u=0 .
$$

In order to pass to the limit as $k$ tends to infinity in the inequality (73), we recall that $H(x, u, \nabla u) \in L^{1}(\Omega)$ and $[a(x)+$ $\left.|u|^{q}\right] \nabla u \in L^{1}(\Omega)$,. We obtain

$$
\begin{gathered}
\int_{\Omega}\left[a(x)+|u|^{q}\right] \nabla u \nabla \psi+\int_{\Omega} u \psi \\
\leq \int_{\Omega} H(x, u, \nabla u) \psi+\int_{\Omega} f \psi
\end{gathered}
$$


for every $\psi \in H_{0}^{1}(\Omega) \cap L^{\infty}(\Omega)$, with $\psi \geq 0$; that is, $u$ is a subsolution of problem (1).

Step 2 (The second inequality). Let $\psi$ be in $H_{0}^{1}(\Omega) \cap L^{\infty}(\Omega)$, with $\psi \leq 0$, and $g$ be given by (58), and choose

$$
\phi=\frac{g^{\rho}(u)}{g^{\rho}\left(u_{n}\right)} R_{k}(u) \psi
$$

asa test function in (16). We obtain

$$
\begin{aligned}
& \int_{\Omega}\left[a(x)+\left|u_{n}\right|^{q}\right] \nabla u_{n} \nabla \psi \frac{g^{\rho}(u)}{g^{\rho}\left(u_{n}\right)} R_{k}(u) \\
& +\rho \int_{\Omega}\left[a(x)+\left|u_{n}\right|^{q}\right] \nabla u_{n} \nabla u \frac{g^{\rho-1}(u)}{g^{\rho}\left(u_{n}\right)} g^{\prime}(u) R_{k}(u) \\
& \cdot \psi+\int_{\Omega}\left[a(x)+\left|u_{n}\right|^{q}\right] \nabla u_{n} \nabla u \frac{g^{\rho}(u)}{g^{\rho}\left(u_{n}\right)} R_{k}^{\prime}(u) \psi \\
& -\rho \int_{\Omega}\left[a(x)+\left|u_{n}\right|^{q}\right] \nabla u_{n} \nabla u_{n} \frac{g^{\rho}(u)}{g^{\rho+1}\left(u_{n}\right)} g^{\prime}\left(u_{n}\right) \\
& \cdot R_{k}(u) \psi-\int_{\Omega} H_{n}\left(x, u_{n}, \nabla u_{n}\right) \frac{g^{\rho}(u)}{g^{\rho}\left(u_{n}\right)} R_{k}(u) \psi \\
& +\int_{\Omega} u_{n} \frac{g^{\rho}(u)}{g^{\rho}\left(u_{n}\right)} R_{k}(u) \psi=\int_{\Omega} f_{n} \frac{g^{\rho}(u)}{g^{\rho}\left(u_{n}\right)} R_{k}(u) \psi
\end{aligned}
$$

We observe that, by (60) and the fact that $\psi \leq 0$, we have

$$
\begin{aligned}
& -\rho\left[a(x)+\left|u_{n}\right|^{q}\right] \nabla u_{n} \nabla u_{n} \frac{g^{\rho}(u)}{g^{\rho+1}\left(u_{n}\right)} g^{\prime}\left(u_{n}\right) R_{k}(u) \psi \\
& -H_{n}\left(x, u_{n}, \nabla u_{n}\right) \frac{g^{\rho}(u)}{g^{\rho}\left(u_{n}\right)} R_{k}(u) \psi \geq-\left(1+\left|u_{n}\right|\right)^{q} \\
& \cdot\left|\nabla u_{n}\right|^{2} \frac{g^{\rho}(u)}{g^{\rho+1}\left(u_{n}\right)} R_{k}(u) \\
& \cdot \psi\left[\tilde{\alpha} \rho g^{\prime}\left(u_{n}\right)-\gamma \frac{g\left(u_{n}\right)}{1+\left|u_{n}\right|}\right] \geq 0 .
\end{aligned}
$$

Applying the same argument of Step 1 and using (64) instead of (63), we deduce that

$$
\begin{array}{r}
\int_{\Omega}\left[a(x)+|u|^{q}\right] \nabla u \nabla \psi+\int_{\Omega} u \psi \\
\leq \int_{\Omega} H(x, u, \nabla u) \psi+\int_{\Omega} f \psi,
\end{array}
$$

for every $\psi \in H_{0}^{1}(\Omega) \cap L^{\infty}(\Omega)$, with $\psi \leq 0$.

Consequently, summarizing Steps 1 and 2, we have

$$
\begin{array}{r}
\int_{\Omega}\left[a(x)+|u|^{q}\right] \nabla u \nabla \psi+\int_{\Omega} u \psi \\
\leq \int_{\Omega} H(x, u, \nabla u) \psi+\int_{\Omega} f \psi,
\end{array}
$$

for every $\psi \in H_{0}^{1}(\Omega) \cap L^{\infty}(\Omega)$.
Finally, interchanging $\psi$ and $-\psi$ we conclude that

$$
\begin{array}{r}
\int_{\Omega}\left[a(x)+|u|^{q}\right] \nabla u \nabla \psi+\int_{\Omega} u \psi \\
=\int_{\Omega} H(x, u, \nabla u) \psi+\int_{\Omega} f \psi,
\end{array}
$$

for every $\psi \in H_{0}^{1}(\Omega) \cap L^{\infty}(\Omega)$.

\section{Data Availability}

The authors do not have data available.

\section{Disclosure}

This paper has been presented at the 5th International Congress of the Moroccan Society of Applied Mathematics (SM2A-2017) and at the second edition of the "Journe doctorale de l'ENSAJ" in the "Ecole Nationale des Sciences Appliques d'El Jadida"- Morocco. We are gratefull to all people who helped in any way in this work.

\section{Conflicts of Interest}

The authors declare that they have no conflicts of interest.

\section{Acknowledgments}

The authors are grateful to all people who helped in this work.

\section{References}

[1] D. Arcoya, L. Boccardo, and T. Leonori, " $W_{0}^{1,1}$-solutions for elliptic problems having gradient quadratic lower order terms," Nonlinear Differential Equations and Applications NoDEA, vol. 20, no. 6, pp. 1741-1757, 2013.

[2] L. Boccardo, F. Murat, and J.-P. Puel, "Existence de solutions non bornées pour certaines équations quasi-linéaires," Portugaliae Mathematica, vol. 41, pp. 507-534, 1982.

[3] A. Bensoussan, L. Boccardo, and F. Murat, "On a nonlinear partial differential equation having natural growth terms and unbounded solution," Ann. Inst. H. Poincaré, vol. 5, no. 4, pp. 347-364, 1988.

[4] L. Boccardo and T. Gallouët, "Strongly nonlinear elliptic equations having natural growth terms and $L^{1}$ data," Nonlinear Analysis: Theory, Methods \& Applications, vol. 19, no. 6, pp. 573579, 1992.

[5] J.-P. Puel, "Existence, comportement à l'infini et stabilité dans certains problmes quasilinéaires elliptiques et paraboliques d'ordre 2," Ann. Scuola Norm. Sup. Pisa Cl. Sci, vol. 3, no. 1, pp. 89-119, 1976.

[6] L. Boccardo, "Dirichlet problems with singular and gradient quadratic lower order terms," ESAIM: Control, Optimisation and Calculus of Variations, vol. 14, no. 3, pp. 411-426, 2008.

[7] L. Boccardo, "A contribution to the theory of quasilinear elliptic equations and application to the minimization of integral functionals," Milan Journal of Mathematics, vol. 79, no. 1, pp. 193-206, 2011. 


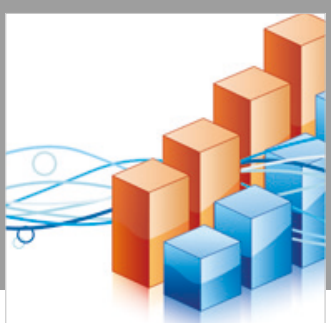

Advances in

Operations Research

\section{-n-m}
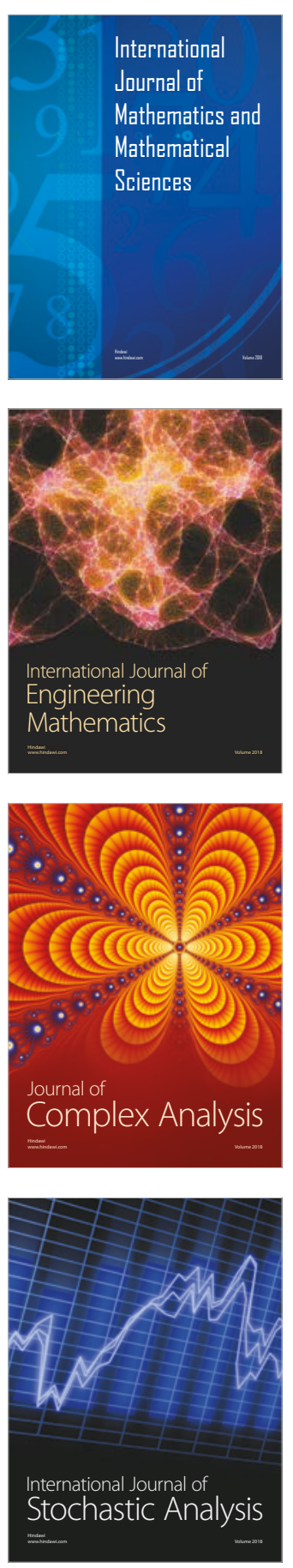
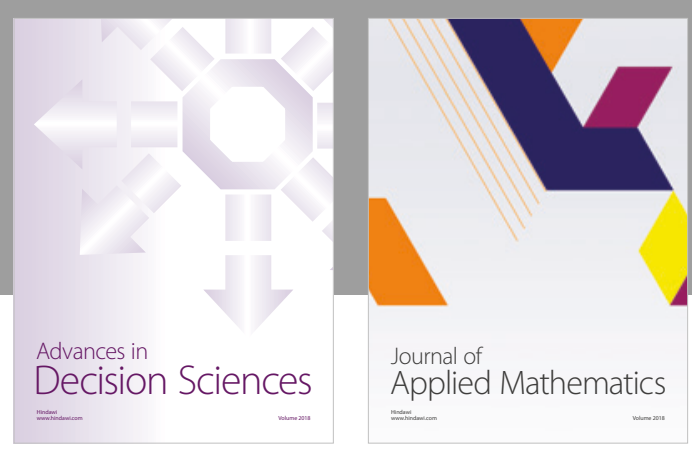

Journal of

Applied Mathematics
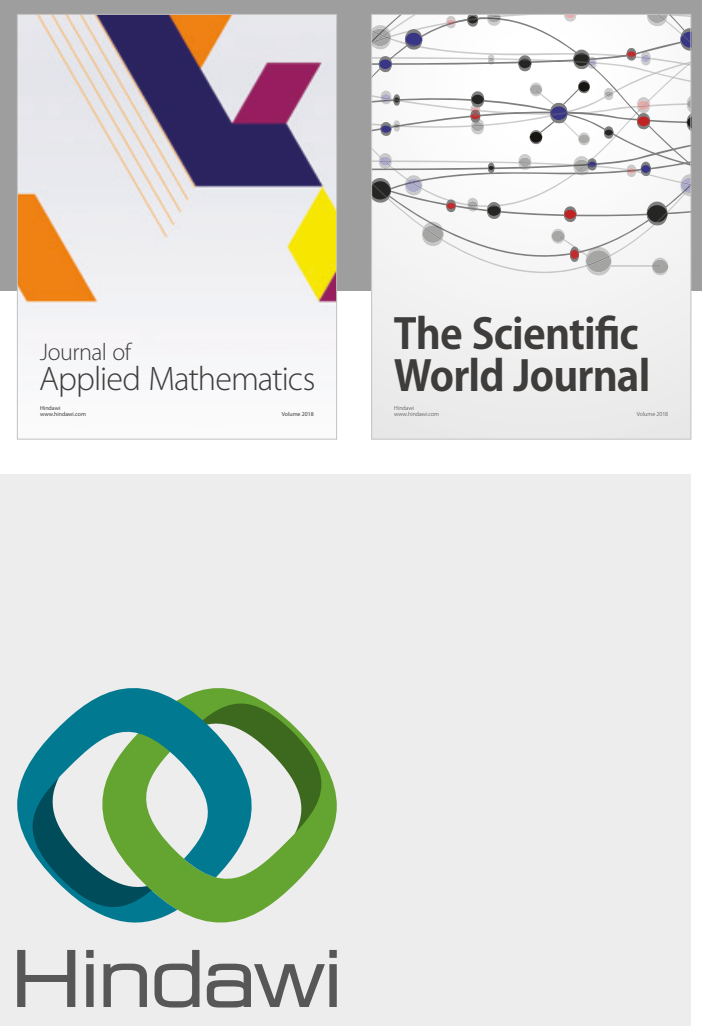

Submit your manuscripts at

www.hindawi.com

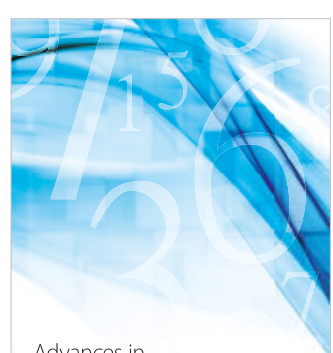

Advances in
Numerical Analysis
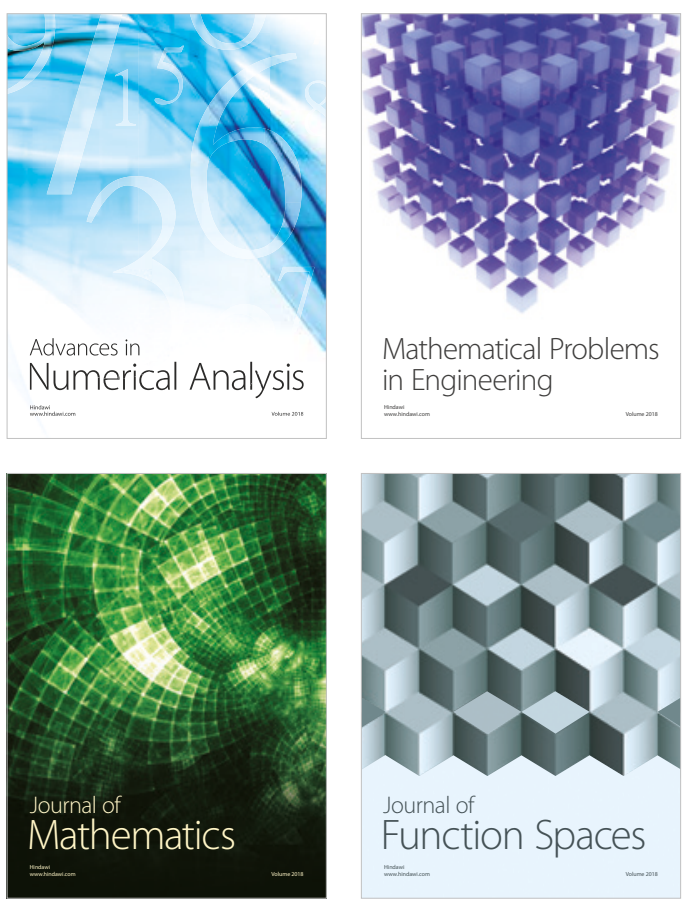

Mathematical Problems in Engineering

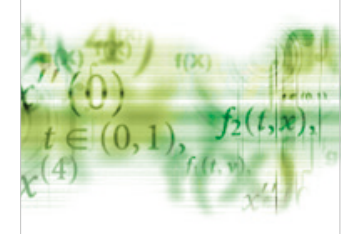

International Journal of

Differential Equations

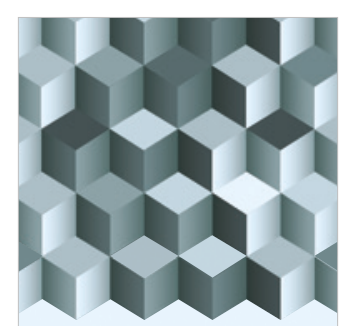

Journal of

Function Spaces

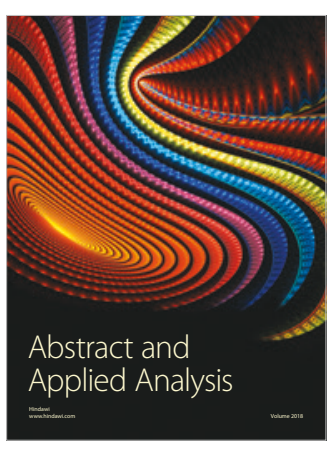

The Scientific

World Journal

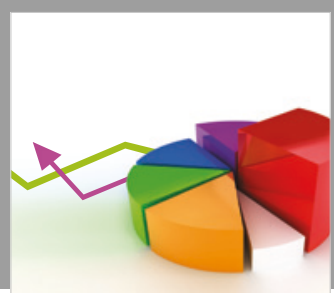

Journal of

Probability and Statistics
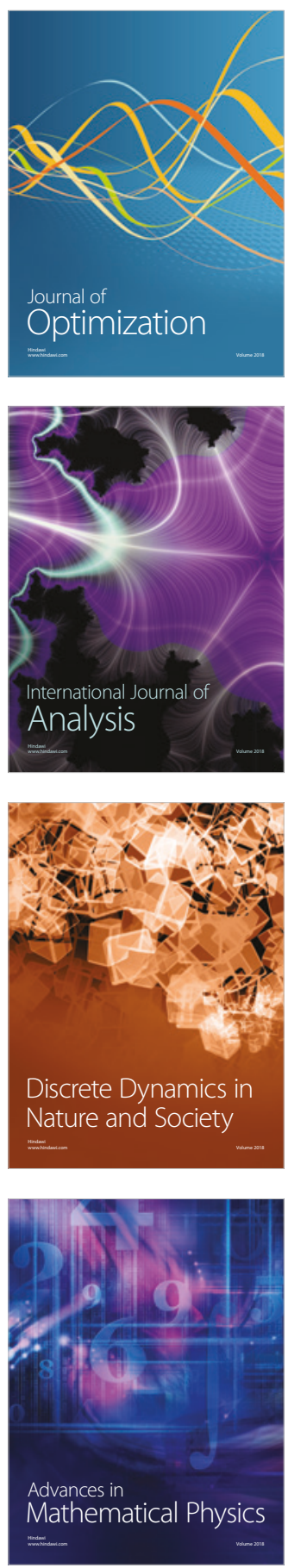